\title{
Correction: Benchmarking Triage Capability of Symptom Checkers Against That of Medical Laypersons: Survey Study
}

Malte L Schmieding ${ }^{1,2}$, MD; Rudolf Mörgeli ${ }^{1}$, MD; Maike A L Schmieding ${ }^{3}$; Markus A Feufel ${ }^{4 *}$, Dipl-Ing (FH), MSc, $\mathrm{PhD}$; Felix Balzer ${ }^{1,2^{*}}$, MSc, PhD, MD

\footnotetext{
${ }^{1}$ Department of Anesthesiology and Operative Intensive Care, Charité - Universitätsmedizin Berlin, corporate member of Freie Universität Berlin and Humboldt-Universität zu Berlin, Berlin, Germany

${ }^{2}$ Institute of Medical Informatics, Charité - Universitätsmedizin Berlin, corporate member of Freie Universität Berlin and Humboldt-Universität zu Berlin, Berlin, Germany

${ }^{3}$ Department of Biology, Chemistry, and Pharmacy, Institute of Pharmacy, Freie Universität Berlin, Berlin, Germany

${ }^{4}$ Department of Psychology and Ergonomics (IPA), Division of Ergonomics, Technische Universität Berlin, Berlin, Germany

*these authors contributed equally
}

\section{Corresponding Author:}

Felix Balzer, MSc, PhD, MD

Institute of Medical Informatics

Charité - Universitätsmedizin Berlin, corporate member of Freie Universität Berlin and Humboldt-Universität zu Berlin

Charitéplatz 1

Berlin, 10117

Germany

Phone: 4930450570425

Email: felix.balzer@charite.de

\section{Related Article:}

Correction of: https://www.jmir.org/2021/3/e24475/

(J Med Internet Res 2021;23(5):e30215) doi: 10.2196/30215

In "Benchmarking Triage Capability of Symptom Checkers Against That of Medical Laypersons: Survey Study" (J Med Internet Res 2021;23(3):e24475) the authors noted two errors.

In the originally published manuscript, Reference 24 in the reference list was cited incorrectly. This citation has now been corrected to the reference listed below [1].
In the originally published paper, there was an error in the phone number of the Corresponding Author. The number has been corrected from "49 304505704 " to "49 30450 570425".

The correction will appear in the online version of the paper on the JMIR Publications website on May 6, 2021, together with the publication of this correction notice. Because this was made after submission to PubMed, PubMed Central, and other full-text repositories, the corrected article has also been resubmitted to those repositories.

\section{Reference}

1. Schmieding ML, Mörgeli R, Schmieding MAL, Feufel MA, Balzer F. Data set supplementing "Benchmarking triage capability of symptom checkers against that of medical laypersons: Survey study". Zenodo. 2021. URL: https://zenodo.org/ record/4454538\#.YJL3Eq1Kj0p [accessed 2021-05-05]

This is a non-peer-reviewed article. Submitted 05.05.21; accepted 05.05.21; published 06.05.21.

Please cite as:

Schmieding $M L$, Mörgeli R, Schmieding MAL, Feufel MA, Balzer F

Correction: Benchmarking Triage Capability of Symptom Checkers Against That of Medical Laypersons: Survey Study

J Med Internet Res 2021;23(5):e30215

URL: https://www.jmir.org/2021/5/e30215

doi: $10.2196 / 30215$

PMID: 
CMalte L Schmieding, Rudolf Mörgeli, Maike A L Schmieding, Markus A Feufel, Felix Balzer. Originally published in the Journal of Medical Internet Research (https://www.jmir.org), 06.05.2021. This is an open-access article distributed under the terms of the Creative Commons Attribution License (https://creativecommons.org/licenses/by/4.0/), which permits unrestricted use, distribution, and reproduction in any medium, provided the original work, first published in the Journal of Medical Internet Research, is properly cited. The complete bibliographic information, a link to the original publication on https://www.jmir.org/, as well as this copyright and license information must be included. 